\title{
Materiales de soporte para el crecimiento de biopelícula en un reactor de lecho fluidizado
}

\section{Advanced support materials for biofilm attachment in a fluidized bed reactor}

\author{
Rubén Alfonso Saucedo-Terán ${ }^{1,5}$, Guadalupe Virginia Nevárez-Moorillón², \\ Raúl Germán Bautista-Margulis ${ }^{3}$, Luisa Idelia Manzanares Papayanopoulos ${ }^{4}$.
}

\section{Resumen}

Se evaluó el efecto de materiales de soporte de baja densidad sobre la eficiencia de un reactor de lecho fluidizado para el tratamiento de aguas residuales. Primero se seleccionaron varios materiales de origen mineral, con densidad específica menor que la densidad de la arena sílica. Después se evaluaron otros materiales porosos de densidad específica ligeramente menor a la densidad de la arena sílica, pero con una densidad aparente significativamente menor. De un total de 10 materiales evaluados, sobresalieron la mezcla de poliéster-perlita expandida y la perlita vitrificada como los mejores materiales. La fluidización del lecho de poliéster-perlita se obtuvo con una velocidad de recirculación de 0.45 y la de perlita vitrificada con $0.55 \mathrm{~cm} \mathrm{seg}^{-1}$. Ambas velocidades de recirculación son menores a las reportadas para materiales de densidad similar. El régimen de fluidización favoreció la operación del reactor en forma continua sin la necesidad de aplicar lavados del lecho y sin llegar a la saturación del mismo.

Palabras clave: Aguas residuales, materia orgánica, fluidización del lecho.

\section{Abstract}

The effect of low density support materials on a fludized bed reactor efficiency was evaluated. Several mineral materials with specific density lower than that exhibited by the silica sand were selected. Moreover, other porous materials with a specific density similar and an appparent density significant lower in comparison to that of the silica sand were also evaluated. We found that the mixture of polyester-expanded perlite and vitrified perlite resulted to be the best as support materials. The bed fluidization for polyester-expanded perlite and vitrified perlite was achieved at a recirculation velocity of 0.45 and $0.55 \mathrm{~cm}$ $\mathrm{seg}^{-1}$, respectively. Both recirculation rates resulted to be lower than those requiered to fluidize similar density materials. These features allowed to the reactor to operate in a continuos mode without needed to wash the bed and avoiding its clogging.

Kwey words: Wastewater, organic matter, bed fluidization.

\footnotetext{
${ }^{1}$ Investigador. Campo Experimental La Campana-Madera. Instituto Nacional de Investigaciones Forestales, Agrícolas y Pecuarias. Ave. Homero No. 3744. Frac. El Vergel. Chihuahua, Chih. C.P. 31100.

${ }^{2}$ Profesora Investigadora. Facultad de Ciencias Químicas. Universidad Autónoma de Chihuahua.

${ }^{3}$ Profesor Investigador. División Académica de Ciencias Biológicas. Universidad Juárez Autónoma de Tabasco.

${ }^{4}$ Directora General de Operación y Control de Auditorías. Procuraduría Federal de Protección al Ambiente. Secretaría del Medio Ambiente y Recursos Naturales.

${ }^{5}$ Dirección electrónica autor de correspondencia: saucedo.ruben@inifap.gob.mx.
}
118
- Vol. II, No. 2 - Mayo-Agosto 2008
TECNOCIENCIAChihuahua 


\footnotetext{
Rubén Alfonso Saucedo-Terán, Guadalupe Virginia Nevárez-Moorillón, Raúl Germán Bautista-

Margulis, luisa Idelia Manzanares Papayanopoulos: Materiales de soporte para el crecimiento de biopelícula en un reactor de lecho fluidizado
}

\section{Introducción}

$\mathrm{E}$

I concepto básico de los reactores de lecho fluidizado consiste en una columna empacada con un lecho de partículas. En dicha columna fluye de manera ascendente cierto volumen de agua residual, a una velocidad suficiente para impartir movimiento o fluidización al lecho (Tsuneda et al., 2002). Conforme el agua residual fluye, densas poblaciones de bacterias se desarrollan en la superficie de las partículas consumiendo los contaminantes biodegradables contenidos en el agua (Karachi et al., 2000).

Una vez fluidizado, el medio provee una vasta superficie de crecimiento bacteriano, generando una concentración de biomasa mayor a la de otros sistemas de tratamiento de aguas residuales (Shieh et al., 1981). La principal ventaja de los lechos fluidizados es que no se saturan por el crecimiento de esta biopelícula. Consecuentemente, se pueden usar partículas de soporte pequeñas, dando una mayor área superficial por unidad de volumen del reactor y pudiendo recibir mayores cargas orgánicas (Trapaso, 1985).

El control de las concentraciones de biopelícula es un aspecto clave en la operación de los reactores de lecho fluidizado (Massol-Deyá et al., 1995; Trinet et al., 1991; Davey y O'Toole, 2000)). La base para el funcionamiento adecuado del sistema es el sostenimiento de la biopelícula bajo condiciones estables (Trinet et al., 1991; Bakke et al., 2002). Cuando se utilizan materiales de soporte de alta densidad, se requieren altas velocidades de fluido como medio para obtener la fluidización del lecho (Jiménez et al., 1990; Ryhner et al., 1988; Trinet et al., 1991). Estas altas tasas de fluido crean un efecto de recorte hidrodinámico lo que conduce a una pérdida excesiva de biopelícula (Fox et al., 1990; Schreyer y Coughlin, 1999; Tavares et al., 1995). En contraparte, cuando se pretende controlar la pérdida de biopelícula mediante la disminución de la velocidad de fluido, se revierte el desprendimiento de biomasa, llegando al extremo en el que el desempeño del reactor corresponde a un lecho fijo, cuya desventaja es la saturación del lecho en menor tiempo (Rivas et al., 2001).

Los materiales de soporte de baja densidad permiten la expansión del lecho hasta en un $100 \%$ bajo tasas de recirculación menores a las requeridas para arena o carbón activado (Trinet et al., 1991; Bignami et al., 1991). Tavares et al., (1995) reportaron el uso de una resina con la que prepararon partículas de $2.7 \mathrm{~mm}$ de diámetro y densidad de $1180 \mathrm{~kg} \mathrm{~m}^{-3}$ resultando fácilmente fluidizables y con buena capacidad de fijación de biopelícula. Un medio de soporte ampliamente utilizado es el carbón activado; no obstante, una desventaja de este material es su excesiva retención de biomasa por lo que su utilización requiere de mecanismos especiales para el desprendimiento de biomasa (Safferman y Bishop, 1996). EI objetivo de este trabajo fue evaluar nuevos materiales de soporte de baja densidad en función de su efecto sobre la eficiencia de un reactor de lecho fluidizado para el tratamiento de aguas residuales.

\section{Materiales y métodos}

El reactor fue hecho de una columna de material acrílico de $8.9 \mathrm{~cm}$ de diámetro interno y $90 \mathrm{~cm}$ de altura. Estas dimensiones son las óptimas para reducir el efecto de pared y la caída de presión (Ocheng et al., 2002; Tsuneda et al., 2002). En la parte superior del reactor fue colocada 
Rubén Alfonso Saucedo-Terán, Guadalupe Virginia Nevárez-Moorillón, Raúl Germán Bautista-

Margulis, Luisa Idelia Manzanares Papayanopoulos: Materiales de soporte para el crecimiento de biopelícula en un reactor de lecho fluidizado

una malla de acero inoxidable de $2 \mathrm{~mm}$ de apertura, cuya función fue la de separar la biomasa e impedir el paso de las partículas de soporte. También se integró un presedimentador para la colecta y purga de lodos, así como un sedimentador para reducir el contenido de sólidos suspendidos en el efluente final. En la parte inferior del sedimentador se colocó un recipiente donde el precipitado del efluente era recirculado con una bomba centrífuga de $1 / 50 \mathrm{cp}$. El flujo se midió con un rotámetro y se controló con una válvula de esfera.

Figura 1. Diagrama del reactor de lecho fluidizado

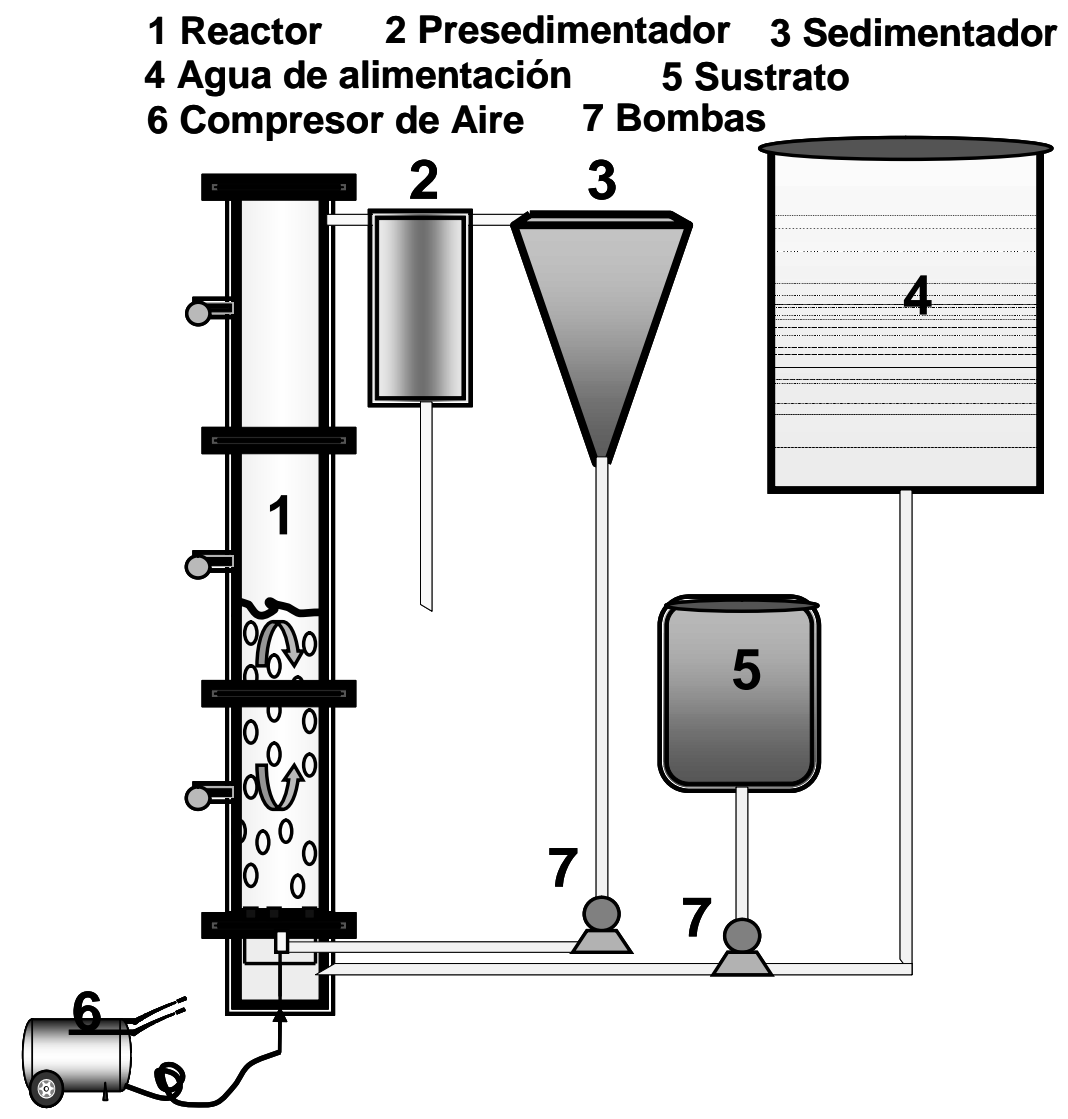

Como sistema de difusión de aire se implementó un plenum de $5 \mathrm{~cm}$ de alto ubicado en la parte inferior del reactor, en el cual se inyectaba el aire procedente de un compresor con un caudal de $1.2 \mathrm{~L} \mathrm{~min}^{-1}$, equivalente a una velocidad de $10 \mathrm{~m} \mathrm{~h}^{-1}$. El aire pasaba a la zona de reacción a través de un disco cerámico poroso. Para unir el disco cerámico al cuerpo del reactor se utilizó una brida de material acrílico de $0.9 \mathrm{~cm}$ de espesor, y de 16.7 y $8.9 \mathrm{~cm}$ de diámetros exterior e interior, respectivamente. Para mantener una temperatura constante en el reactor, se empleó un calentador eléctrico de 100 watts y un termostato. Como sustituto de aguas residuales se preparó un substrato concentrado compuesto de dextrosa, glicerol, urea y $\mathrm{KH}_{2} \mathrm{PO}_{4}$ y extracto de carne. Todos los ingredientes eran de grado reactivo, excepto el extracto de carne.

Con el fin de obtener los materiales de soporte con las características deseadas de 


\begin{abstract}
Rubén Alfonso Saucedo-Terán, Guadalupe Virginia Nevárez-Moorillón, Raúl Germán BautistaMargulis, Luisa Idelia Manzanares Papayanopoulos: Materiales de soporte para el crecimiento de biopelícula en un reactor de lecho fluidizado
\end{abstract}

densidad, porosidad y resistencia mecánica, se procedió a elaborar una lista de candidatos de entre varias opciones de materiales de origen mineral, teniendo como principal característica una densidad específica menor que la densidad de la arena sílica. En una segunda etapa, se evaluaron varios materiales porosos de densidad aparente menor a la densidad de la arena sílica. En este caso, se planteó hipotéticamente que algunos materiales de densidad aparente baja podrían ser fluidizados bajo velocidades de recirculación menores a los requeridos para fluidizar materiales de densidad específica alta. Esta hipótesis se basó en la presunción de que la presencia de la biopelícula evitaría que los poros internos de los materiales se mantuvieran ocupados por aire, evitando que éste fuera desplazado por el agua $y$, por lo tanto, conservarían una fuerza gravitacional menor a la correspondiente al material saturado con agua.

La perlita es un mineral abundante en nuestro país, el cual al ser sometido a un proceso térmico $\left(800^{\circ} \mathrm{C}\right)$ se expande formando partículas sumamente porosas y ligeras. Una desventaja de la perlita expandida es su baja estabilidad mecánica. No obstante, durante el proceso térmico de expansión de la perlita, se produce una escoria llamada perlita vitrificada, la cual conserva las propiedades de baja densidad pero su tenacidad y dureza se incrementan considerablemente. Por tales razones, la perlita vitrificada también fue considerada como posible material de soporte.

Cuadro 1. Principales características de los materiales preseleccionados como candidatos a medios de soporte

\begin{tabular}{lccc}
\hline Material & \multicolumn{2}{c}{ Densidad $\left(\mathrm{g} / \mathrm{cm}^{3}\right)$} & Diámetro \\
& Especifica & Aparente & $(\mathrm{mm})$ \\
\hline Poliestireno & 1.08 & 0.7060 & $0.7-1.1$ \\
Flaggstafita & 1.09 & - & - \\
Poliéster+perlita & 1.22 & 0.6469 & $2.5-4.00$ \\
Carnalita & 1.60 & - & - \\
Perlita expandida & 1.76 & 0.1231 & Agregados \\
Block espuma & 1.97 & 0.3184 & $2.38-3.36$ \\
Piedra pómez & 2.07 & 0.3173 & $2.38-3.36$ \\
Perlita vitrificada & 1.71 & 0.4860 & $2.10-4.00$ \\
Pumicita & 2.31 & 0.6287 & $2.38-3.36$ \\
Basalto CJ & 2.45 & 0.8068 & $2.38-3.36$ \\
Arena sílica & 2.60 & 1.6145 & $2.38-3.36$ \\
Alúmina & 2.76 & 0.8092 & $2.38-3.36$ \\
\hline
\end{tabular}


Rubén Alfonso Saucedo-Terán, Guadalupe Virginia Nevárez-Moorillón, Raúl Germán BautistaMargulis, Luisa Idelia Manzanares Papayanopoulos: Materiales de soporte para el crecimiento de biopelícula en un reactor de lecho fluidizado

También se incluyeron dos materiales poliméricos de baja densidad. Una resina de intercambio iónico de partículas de poliestireno y una resina de poliéster, la cual fue mezclada con la perlita expandida a fin de mejorar sus propiedades de porosidad y rugosidad superficial. En el Cuadro 1 se muestran las principales características de los medios de soporte evaluados, entre las que destacan las bajas densidades de la resina de poliestireno $\left(1.08 \mathrm{~g} \mathrm{~cm}^{-3}\right)$, de la flaggstafita $\left(1.09 \mathrm{~g} \mathrm{~cm}^{-3}\right)$ y de la mezcla de poliéster + perlita $\left(1.22 \mathrm{~g} \mathrm{~cm}^{-3}\right)$.

A diferencia del resto de los materiales, la mezcla de poliéster con perlita expandida y la perlita vitrificada requirieron de un proceso de preparación, antes de ser utilizados en el reactor. El poliéster, el cual se expende comercialmente en estado líquido y cuya densidad es de $1.008 \mathrm{~g} \mathrm{~cm}^{-3}$, se mezcló con un 50\% (volumen/volumen aparente) de perlita expandida (Carlita M.R.) con densidad de $1.76 \mathrm{~g} \mathrm{~cm}^{-3}$. A la mezcla se agregó 2\% (v/v) de etil metil cetona como catalizador para obtener su solidificación, siendo inmediatamente vaciada en un recipiente de acero inoxidable. Cuando el poliéster presentaba un estado gelatinoso se procedió a realizar una serie de cortes con navaja, generando cubos de alrededor de $0.5 \mathrm{~cm}$ por lado. Una vez que la mezcla se solidificó por completo, los cubos fueron sometidos a un proceso de esferonización. La perlita vitrificada también fue sometida a esferonización, después de ser triturada hasta obtener partículas de aproximadamente 5 a $8 \mathrm{~mm}$ de diámetro. La esferonización se efectuó colocando los materiales en un recipiente metálico cuyo fondo estaba constituido por un disco abrasivo rotatorio, el cual era accionado por un motor eléctrico de $0.5 \mathrm{cp}$. La forma esférica de las partículas se obtenía por efecto de la fricción causada por la rotación del disco abrasivo a una velocidad de 600 rpm

Cuadro 2. Materiales de soporte y concentración de la demanda química de oxígeno (DQO) del influente en las corridas experimentales efectuadas.

\begin{tabular}{cccc}
\hline $\begin{array}{c}\text { No. de } \\
\text { Corrida }\end{array}$ & $\begin{array}{c}\text { Duración } \\
\text { (dias) }\end{array}$ & $\begin{array}{c}\text { Material de } \\
\text { Soporte }\end{array}$ & $\begin{array}{c}\text { Concentración de } \\
\text { DQO (mgíL) }\end{array}$ \\
\hline A1 & 5 & Poliestireno & 210 \\
A2 & 7 & Alúmina activada & 300 \\
B1, B2 & 41 & Poliéster + perlita & 210,280 y 350 \\
C1, C2 & 21 & Poliéster + perlita & 210 \\
D1, D2 & & & \\
C3-C5 & 21 & Perlita vitrificada & 210 \\
D2, D3 & & & \\
\hline
\end{tabular}




\begin{abstract}
Rubén Alfonso Saucedo-Terán, Guadalupe Virginia Nevárez-Moorillón, Raúl Germán BautistaMargulis, Luisa Idelia Manzanares Papayanopoulos: Materiales de soporte para el crecimiento de biopelícula en un reactor de lecho fluidizado
\end{abstract}

Para evaluar la eficiencia del reactor, se llevaron a cabo 9 corridas experimentales (Cuadro 2). Dos corridas cortas $(A 1, A 2)$ de cinco y siete días de duración, respectivamente, en las que se evaluaron los materiales de poliestireno y de alúmina activada. Dos corridas largas (B1, B2) de 41 días de duración cada una en las que se empleó la mezcla de poliéster y perlita expandida. Nueve corridas de 21 días de duración, cuatro corridas con poliéster + perlita (C1, C2; D1, D2) y cinco corridas con perlita vitrificada (C3-C5; D2, D3), en las que conjuntamente con los resultados de los primeros 21 días de las corridas B1 y B2 se estudió la dinámica de diferentes indicadores de crecimiento de la biopelícula y su relación con la eficiencia del sistema. Estos datos se analizaron bajo un diseño aleatorio simple y un arreglo de mediciones repetidas. Las condiciones de operación de las corridas experimentales se muestran en el Cuadro 3.

Cuadro 3. Condiciones de operación en las corridas experimentales con los materiales de soporte a base de poliéster+perlita y perlita vitrificada

\begin{tabular}{lcc}
\hline Variable & Poliéster + Perlita & Perlita vitrificada \\
\hline Tiempo de retención hidráulica $(\mathrm{min})$ & 30 & 30 \\
Altura estática del lecho $(\mathrm{cm})$ & 30 & 30 \\
Volumen del lecho $(\mathrm{L})$ & 2.450 & 2.450 \\
Flujo de recirculación $\left(\mathrm{L} \mathrm{min}^{-1}\right)$ & 1.7 & 2.1 \\
Velocidad de recirculación $\left(\mathrm{cm} \mathrm{seg}^{-1}\right)$ & 0.45 & 0.55 \\
Flujo de influente $(\mathrm{L} \mathrm{min-1})$ & 0.14 & 0.14 \\
Velocidad superficial del aire $\left(\mathrm{m} \mathrm{h}^{-1}\right)$ & 10 & 10 \\
\hline
\end{tabular}

La determinación de la concentración de microorganismos en el medio de soporte se llevó a cabo mediante el monitoreo de la concentración de proteínas y polisacáridos, principales componentes bioquímicos indicadores del crecimiento de la biopelicula (Characklis et al., 1982). Como indicadores de condiciones de operación y de calidad del efluente se monitorearon los cambios en las concentraciones de demanda química de oxígeno (DQO), de acuerdo con la técnica normalizada de oxidación de dicromato de potasio (Ramalho, 1996).

Resultados y Discusión

En la primera etapa de selección de materiales se eliminaron candidatos como la flaggstafita y la carnalita. A pesar de tener densidades específicas de 1.09 y $1.6 \mathrm{~g} \mathrm{~cm}^{-}$ ${ }^{3}$, no obstante, son materiales son difíciles de obtener y por lo tanto, de alto costo. También se eliminaron la perlita expandida, el block espuma, la piedra pómez y la 
Rubén Alfonso Saucedo-Terán, Guadalupe Virginia Nevárez-Moorillón, Raúl Germán BautistaMargulis, Luisa Idelia Manzanares Papayanopoulos: Materiales de soporte para el crecimiento de biopelícula en un reactor de lecho fluidizado

pumicita, materiales de fácil fluidización, pero de baja estabilidad mecánica. Así mismo, se eliminó el basalto volcánico, material de baja densidad aparente pero de rápida saturación con agua por lo que sus requerimientos de fluidización rebasaron los límites establecidos en este trabajo (Cuadros 4 y 5).

Cuadro 4. Resultados generales de los candidatos para materiales de soporte seleccionados en este trabajo.

\begin{tabular}{|c|c|}
\hline Material & Observaciones Generales \\
\hline Poliestireno & $\begin{array}{l}\text { Fácilmente fluidizable } \\
\mathrm{U}_{\mathrm{mf}}^{*}=0.037 \mathrm{~cm} \mathrm{seg}^{-1}\end{array}$ \\
\hline Poliéster + perlita & $\begin{array}{l}\text { Fácilmente fluidizable } \\
U_{\mathrm{mf}}=0.35 \mathrm{~cm} \mathrm{seg}^{-1}\end{array}$ \\
\hline \multicolumn{2}{|l|}{ Perlita expandida } \\
\hline Block espuma & Baja estabilidad mecánica \\
\hline \multicolumn{2}{|l|}{ Piedra pómez } \\
\hline Perlita vitrificada & $\begin{array}{l}\text { Fácilmente fluidizable } \\
U_{\mathrm{mf}}=0.45 \mathrm{~cm} \mathrm{seg}^{-1}\end{array}$ \\
\hline Pumicita & Soluble en agua \\
\hline $\begin{array}{l}\text { Basalto CJ } \\
\text { Arena sillica }\end{array}$ & $\begin{array}{l}\text { Fluidización bajo velocidades de } \\
\text { recirculación por encima de los límites }\end{array}$ \\
\hline Alúmina & preestablecidos en este trabajo \\
\hline
\end{tabular}

En la segunda fase de selección se eliminaron la resina de poliestireno y la alúmina activada. En el caso de la resina de poliestireno se obtuvo una fluidización totalmente libre de turbulencia, sin la necesidad de aplicar el flujo de recirculación, alcanzando una expansión del lecho superior al $100 \%$. No obstante, debido al escaso movimiento de las partículas y la poca fricción entre ellas, a partir del segundo día de operación se observaron coágulos formados por la biomasa con las partículas del lecho, siendo prácticamente imposible su desintegración, sin causar el arrastre y la expulsión de biopartículas hacia el exterior del reactor (Cuadro 5). 
Rubén Alfonso Saucedo-Terán, Guadalupe Virginia Nevárez-Moorillón, Raúl Germán BautistaMargulis, Luisa Idelia Manzanares Papayanopulos: Materiales de soporte para el crecimiento de biopelícula en un reactor de lecho fluidizado

Cuadro 5. Resumen de resultados del proceso de selección de materiales de soporte en el total de corridas experimentales efectuadas

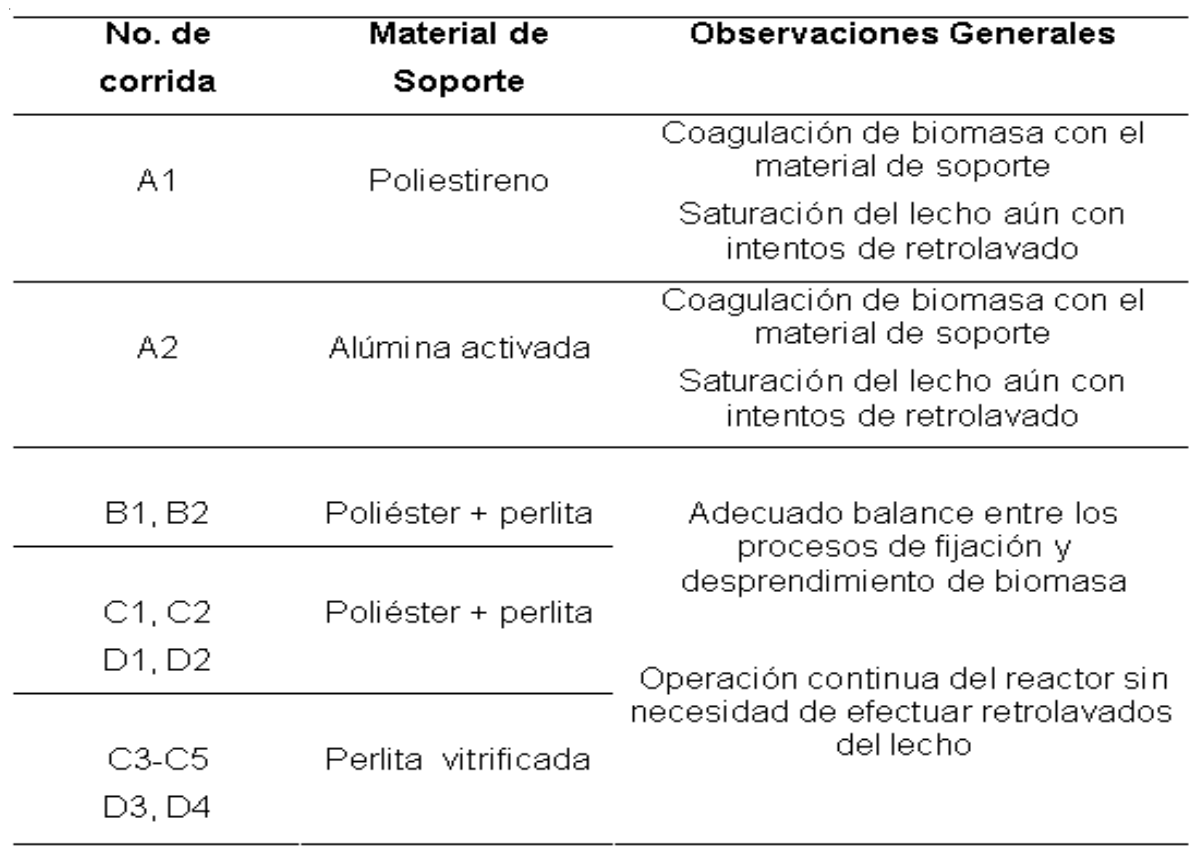

En el quinto día de operación, el total del lecho formó con la biomasa una sola pieza gelatinosa, la cual no fue posible desprender aún con el incremento de la velocidad de flujo de las fases líquida y gaseosa. Además de la coagulación, también se presentó acanalamiento de substrato y aire, terminando finalmente en el estancamiento del lecho, formándose abundantes zonas anóxicas consistentes en corpúsculos de color negro y fuerte olor a compuestos azufrados.

En el caso de la alúmina, al término de los 7 días de prueba, se observó la presencia de una biopelícula densa y uniforme alrededor de las partículas del material de soporte. A pesar de incrementar los regímenes de velocidad del flujo de agua y aire, el lecho en su totalidad no pudo ser fluidizado. Sólo se obtuvo la fluidización de aproximadamente el $10 \%$ de la parte superior del lecho, bajo un flujo de recirculación de $3,000 \mathrm{~cm}^{3} \mathrm{seg}^{-1}$ equivalente a una velocidad de recirculación de $0.80 \mathrm{~cm} \mathrm{seg}^{-1}$, muy próximo al límite de flujo de recirculación considerado en este trabajo. Sin embargo, al permanecer fijo el resto del lecho, éste se empezó a coagular con la biomasa terminando por estancarse y formar una sola pieza gelatinosa, la cual también fue imposible de disgregar mediante el incremento en la velocidad del aire (Cuadro 5).

El desempeño de la alúmina se puede entender por el hecho de que su densidad es similar a la de la arena sílica, la que bajo condiciones de poca o nula fluidización, se comporta como un lecho fijo, terminando por saturarse en poco tiempo (Rivas et al., 2001). A pesar de lo anterior, la alúmina fue incluida en este trabajo porque se planteó la posibilidad de que, dada su baja densidad aparente podría ser fluidizada bajo velocidades de recirculación menores a los requeridos para fluidizar materiales de densidad específica alta. Sin embargo, para 
Rubén Alfonso Saucedo-Terán, Guadalupe Virginia Nevárez-Moorillón, Raúl Germán BautistaMargulis, luisa Idelia Manzanares Papayanopoulos: Materiales de soporte para el crecimiento de biopelícula en un reactor de lecho fluidizado

que lo anterior se cumpliera, era necesario que los poros internos de las partículas de alúmina no fueran saturados con agua, ya que se forman pequeñas cámaras de aire, que se podrían evitar si la película de polisacáridos formara una capa impermeable alrededor de las partículas.

La baja densidad de la mezcla de polyester+perlita y de la perlita vitrificada permitió que ambos materiales fueran fácilmente fluidizables, aún mediante velocidades de recirculación por debajo de las reportadas en otros estudios para materiales de soporte de baja densidad (Tavares et al., 1995). La fluidización del lecho se llevó a cabo por efecto de la recirculación de la fase líquida, de acuerdo con el diseño de Larachi et al., (2000). Partiendo de flujos de recirculación mayores al inicio de la operación del reactor, conforme la densidad de las partículas del lecho cambiaba por efecto de la adhesión de biopelícula. El flujo de recirculación fue gradualmente ajustado hasta un flujo final de $1.7 \mathrm{~cm}^{-3} \mathrm{~min}^{-1}$ para el lecho de poliéster y de $2.1 \mathrm{~cm}^{-3} \mathrm{~min}^{-1}$ para el de perlita vitrificada, equivalentes a velocidades de recirculación de 0.45 y $0.55 \mathrm{~cm} \mathrm{seg}^{-1}$, respectivamente. De acuerdo con lo expresado por Larachi et al., (2000) estas velocidades de recirculación resultaron teóricamente mayores a la mínima velocidad de fluidización, ya que debido a la caída de presión en el reactor, la mínima velocidad de fluidización para las partículas de poliéster+ perlita y para las de perlita vitrificada fue de $0.35 \mathrm{~cm} \mathrm{seg}^{-1} y$ de $0.45 \mathrm{~cm}$ $\mathrm{seg}^{-1}$, respectivamente (Figura 2 ).

La fluidización del lecho ejerció un papel fundamental en la prevención del

Figura 2. Comportamiento del lecho en un reactor trifásico de lecho fluidizado, bajo diferentes velocidades de recirculación de la fase líquida. Las flechas indican la zona de fluidización.

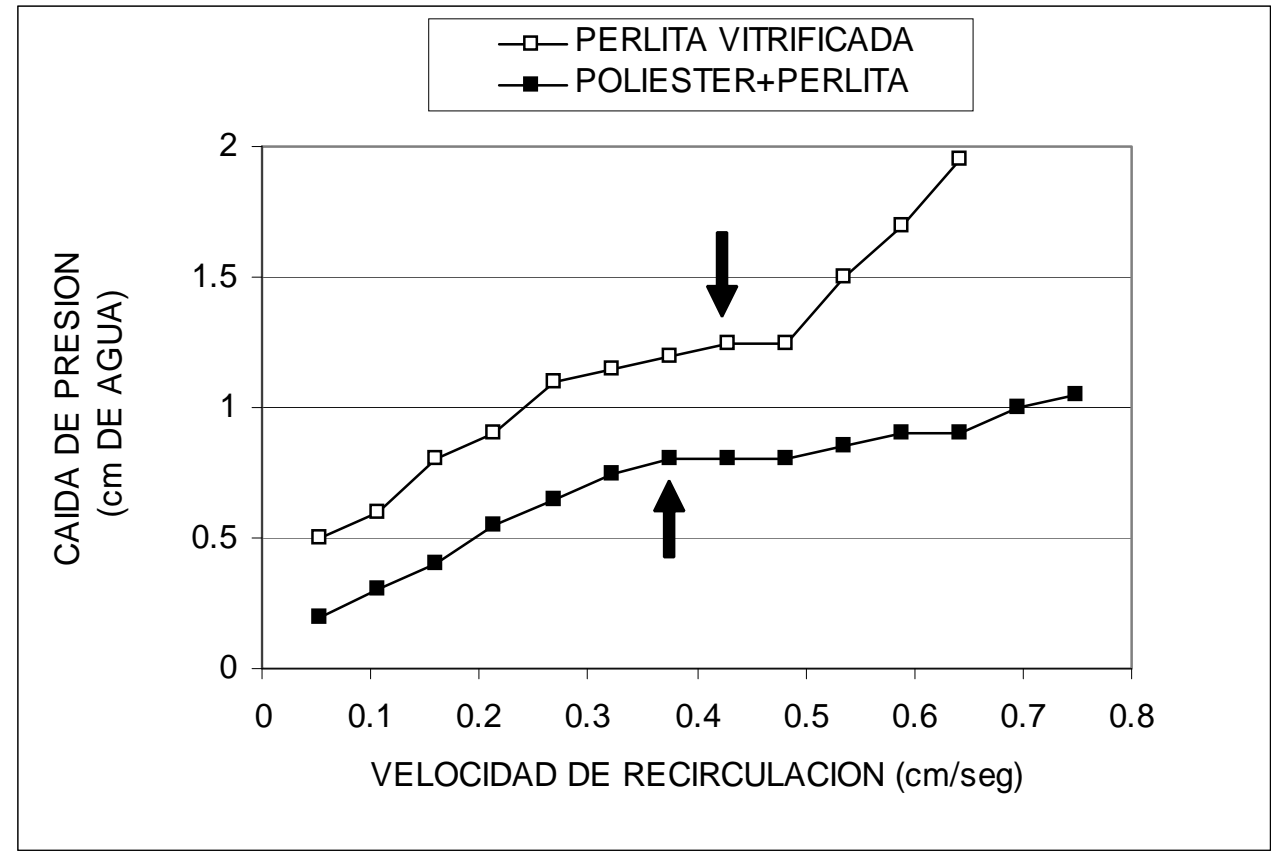

Comportamiento del lecho en un reactor trifásico de lecho fluidizado, bajo diferentes velocidades de recirculación de la fase líquida. Las flechas indican la zona de fluidización. 
Rubén Alfonso Saucedo-Terán, Guadalupe Virginia Nevárez-Moorillón, Raúl Germán Bautista-

Margulis, Luisa Idelia Manzanares Papayanopoulos: Materiales de soporte para el crecimiento de biopelícula en un reactor de lecho fluidizado

estancamiento del reactor, dado que propició un movimiento de las biopartículas con la suficiente fuerza de arrastre requerida para evitar la aglutinación y finalmente la coagulación del lecho por la adhesión de biomasa. La fluidización obtenida consistió en un flujo ascendente por el centro del reactor en el que las biopartículas al llegar a la zona de separación de biomasa, ubicada en la parte superior del reactor, perdían parte de su biopelícula por acción de la turbulencia creada por las burbujas de aire así como por su fricción contra las paredes de la malla de acero.

Sin embargo, de acuerdo a los resultados de Nicolella et al., (1997), la abrasión causada por la colisión entre partículas es considerada como el mecanismo clave para el desprendimiento del exceso de biopelícula en el sistema de tratamiento de aguas residuales bajo estudio. Al perder parte de su biopelícula, la densidad de las partículas se veía aumentada por lo que iniciaban un flujo descendente, deslizándose por las paredes hasta llegar al fondo del reactor. Desde ahí se reiniciaba el flujo ascendente por un efecto sinérgico de arrastre causado por los flujos de recirculación, del sustrato y de las burbujas de aire.

Desde el primer día de operación, se observó una importante actividad microbiológica, en términos de cantidad de biopelícula como en la disminución del contenido de DQO en el efluente, independientemente del material de soporte empleado (Figura 3). A pesar de que durante el transcurso del trabajo se observaron importantes variaciones en las concentraciones de proteínas y carbohidratos, estos indicadores mostraron cierta estabilidad, situándose en su mayoría entre 30 y $40 \mathrm{mg}$ y entre 20 y $25 \mathrm{mg} \mathrm{g}^{-1} \mathrm{de}$ material de soporte, respectivamente La estabilización de la biopelícula ocurre generalmente alrededor de los 21 días de operación (Davey y O'Toole; Blake et al., 2001).

La figura 4 muestra la remoción de la demanda química de oxigeno en seis corridas con poliéster+perlita y cinco con perlita vitrificada como material de soporte. Ambos materiales mostraron niveles de

Figura 3. Componentes microbianos como indicadores de crecimiento biopelicular en un reactor de lecho fluidizado. Los valores son las medias \pm error estándar de 7 corridas (B1, B2; C1, C2; C3-C5).

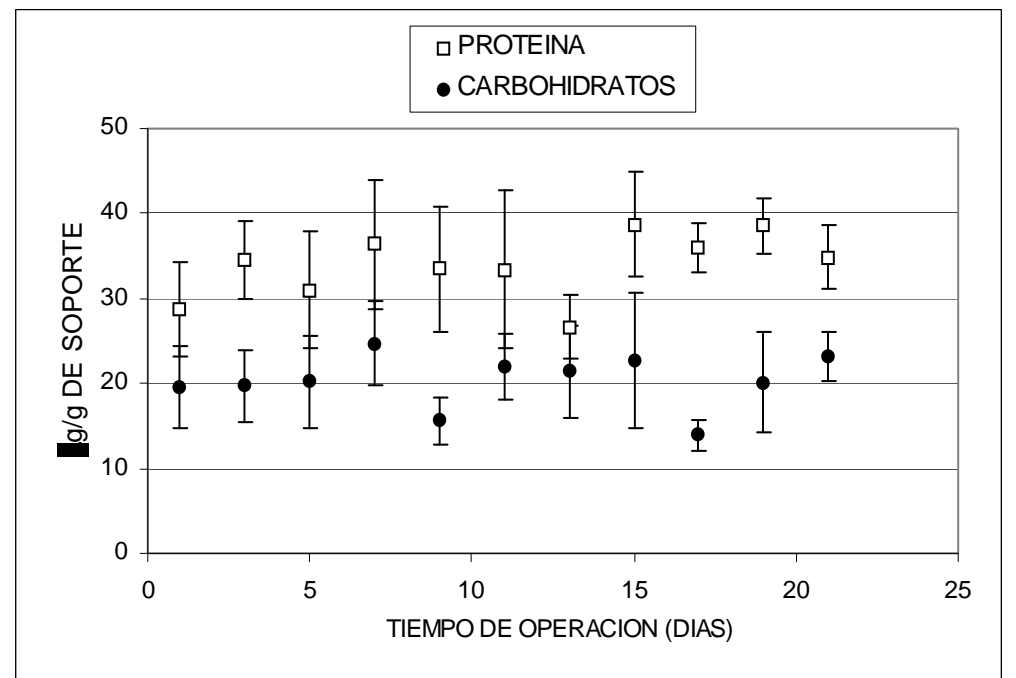


eficiencia similares, resultando ligeramente superior la mezcla de poliéster+perlita hacia el final del periodo de estudio, aunque estadísticamente no se encontraron diferencias $(P>0.05)$ entre materiales de soporte ni en su interacción con el tiempo de operación.

También puede observarse que desde el primer día de operaciones se logró remover alrededor del $60 \%$ de la DQO en ambos materiales. La eficiencia en el reactor empacado con poliéster+perlita mostró niveles de eficiencia de alrededor del 65\% durante los primeros 13 días de operación, incrementándose ligeramente a partir del $13^{\circ}$ día a partir del cual el promedio de remoción de $\mathrm{DQO}$ fue de alrededor de $80 \%$. En cuanto a la perlita vitrificada, su eficiencia se incrementó ligeramente con respecto al primer día de operación, mostrando enseguida una estabilidad con niveles de remoción similares a lo largo del estudio, teniendo un promedio aproximado de $70 \%$ de remoción de DQO (Figura 4).

Figura 4. Eficiencia en la remoción de $\mathrm{DQO}$ en un reactor trifásico de lecho fluidizado con dos materiales de soporte. Los valores son las medias \pm error estándar de 6 corridas con Poliéster+perlita (B1, B2; C1, C2;

D1, D2) y 5 corridas con perlita vitrificada (C3-C5; D3, D4).

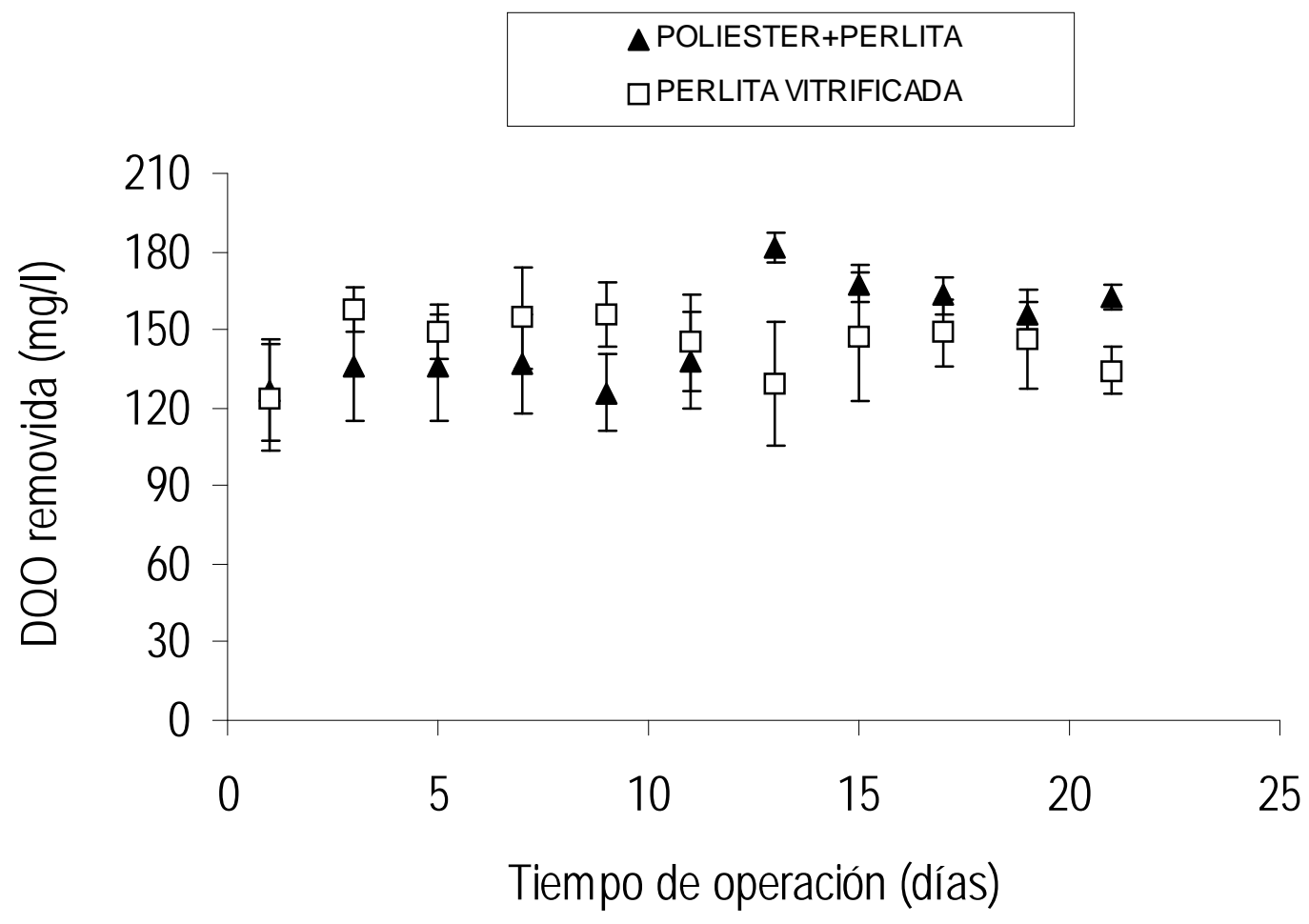


Rubén Alfonso Saucedo-Terán, Guadalupe Virginia Nevárez-Moorillón, Raúl Germán BautistaMargulis, Luisa Idelia Manzanares Papayanopoulos: Materiales de soporte para el crecimiento de biopelícula en un reactor de lecho fluidizado

\section{Conclusiones}

Los medios de soporte de poliéster + perlita y de perlita vitrificada resultaron ser los más eficientes para la fijación de microorganismos degradadores de materia orgánica de un total de diez materiales evaluados.

Las bajas densidades de estos materiales favorecieron la fluidización del lecho con velocidades de recirculación de la fase líquida por debajo de las requeridas para la fluidización de la arena sílica o el carbón activado y aún para materiales de densidad similar a las del presente estudio.

El régimen de fluidización obtenido propició la colisión entre las partículas del lecho, resultando el mecanismo clave para el desprendimiento de la biopelícula en los dos materiales de soporte.

\section{Literatura citada}

BAKKE, R., R. Kommendal S., and S. Kalvenes. 2001. Quantification of Biofilm Accumulation by an Optical Approach. Journal of Microbiological Methods. 44: 13-26.

Bıgnami L., Eramo B., Gavasci R., Ramadori R. and Rolle E. 1991. Modelling and experiments on fluidized-bed biofilm reactors. Water Science and Technology 24: 47-58.

DAVEY, M.E., and G.A. O'Toole. 2000. Microbial Biofilms: from Ecology to Molecular Genetics. Microbiological And Molecular Reviews. 64 (4): 847-867.

Fox, P., M.T. Suidan, and J.T. Bandy. 1990. A Comparison of Media Types in Acetate Fed Expanded-Bed Anaerobic Reactors. Water Research 24 (7): 827-835.

JIMÉNEZ, B., E. Becerril, and I. Scola. 1990. Denitrification in a Fluidized Bed System Using Low Cost Packing Material. Environmental Technology 11: 409-420.

LARACHI, F., I. lliut, O. Rival, and B.P.A. Grandjean. 2000. Prediction of Minimum Velocity in Three- Phase Fluidized-Bed Reactors. Industrial Engineering Chemistry Research. 39 (2): 563572 .

Massol_Deyá, A.D., J. Whallon, R.F. Hickey, and J.M. Tiedje. 1995. Channel Structures in Aerobic Biofilms of Fixed-Film Reactors Treating Contaminated Groundwater. Applied and Environmental Microbiology 61(2): 769-777.

Nicolella C., Chiarle S., Di Felice R. and Rovatti M. 1997. Mechanisms of biofilm detachment in fluidized bed reactors. Water Science and Technology 36: 229-235.

Ochieng, A., T. Ogada, W. Sisenda, and P. Wambua. 2002. Brewery wastewater treatment in a fluidized bed bioreactor. Journal of Hazardous Materials. 90: 311-321.

RIvas, L.B.A., G.V. Nevárez M., R.A. Saucedo T., and R.G. Bautista M. 2000. Simultaneous Aerobic and Anaerobic Degradation of Wastewater in a Fixed-Bed Reactor. En: Ex situ Biological Treatment Technologies. Proceedings of the Sixth International Symposium of In Situ and On-Site Bioremediation. Batelle Press. San Diego, CA. Vol. 6, pp. 361-368.

RAmALHo, R.S. 1996. Tratamiento de Aguas Residuales. Versión en Español por D.J. Beltrán, F. De Lora y R.S. Ramalho. Editorial Reverté. Barcelona, España. 705 p.

RYHNER G., S. Petrozzi, and I.J. Dunn. 1988. Operation of a ThreePhase Biofilm Fluidized Sand Reactor for Aerobic Wastewater Treatment. Biotechnology and Bioengineering 32: 677-688.

Safferman, S.I., and P.L. Bishop. 1996. Aerobic Fluidized Bed Reactor With Internal Media Cleaning. Journal of Environmental Engineering 122 (4): 284-291.

SCHREYER, H.B., and R.W. Coughlin. 1999. Effects of Stratification in a Fluidized Bed Bioreactor During Treatment of Metalworking Wastewater. Biotechnology and Bioengineering 63 (2):129140.

SHIEH, W. K., P. M. Sutton, and P. Kos. 1981. Predicting reactor biomass concentration in a fluidized-bed system. Journal of Water Pollution Control Federation 53: 1574-1584.

Tavares, C.R.G., Sant'Anna, G.L., Jr., Capdeville, B. 1995. The Effect of Air Superficial Velocity on Biofilm Accumulation in a Three-Phase Fluidized-Bed Reactor. Water Research 29 (10): 2293-2298.

TRAPASO, R.G.A. 1995. The optimal design of fluidized bed bioreactors. Journal of Water Pollution Control Federation $57: 143-150$

Tsuneda, S., J. Auresenia, Y. Inoue, Y. Hashimoto, and A. Hirata. 2002. Kinetic model for dynamic response of three-phase fluidized bed biofilm reactor for wastewater treatment. Biochemical Engineering Journal. 10: 31-37. (9)

Este artículo es citado así:

SAUCEDO-Terán Rubén Alfonso, Guadalupe Virginia Nevárez-Moorillón, Raúl Germán Bautista-Margulis, Luisa Idelia Manzanares Papayanopulos. 2008. Materiales de soporte para el crecimiento de biopelícula en un reactor de lecho fluidizado. TECNOCIENCIA Chihuahua 2(2): 40-55 
Rubén Alfonso Saucedo-Terán, Guadalupe Virginia Nevárez-Moorillón, Raúl Germán BautistaMargulis, Luisa Idelia Manzanares Papayanopoulos: Materiales de soporte para el crecimiento de biopelícula en un reactor de lecho fluidizado

\section{Resúmenes curriculares de autor y coautores}

Ruben Alfonso Saucedo Teran. Realizó sus estudios de licenciatura en la Facultad de Zootecnia (FZ) de la Universidad Autónoma de Chihuahua (UACH), obteniendo en 1982 el título de Ingeniero Zootecnista. En el año 1991 le fue otorgado el grado de Maestro en Ciencias, con especialidad en Manejo de Pastizales y Ecología, por la FZ de la UACH. Obtuvo en 2002 su Doctorado en Ciencias, especialidad en Ciencias Ambientales, grado conferido por el Centro de Investigación en Materiales Avanzados (CIMAV) con sede en la ciudad de Chihuahua, México. Desde el año 1981 trabaja como Investigador de Tiempo Completo en el Instituto Nacional de Investigaciones Forestales, Agrícolas y Pecuarias (INIFAP), institución donde realiza investigación sobre Medio Ambiente y Desarrollo Sustentable.

Guadalupe Virginia Nevárez Moorillón. Cursó su licenciatura en la Facultad de Ciencias Químicas de la Universidad Autónoma de Chihuahua (UACH), recibiendo en 1987 el título de Químico Biólogo Parasitólogo con la defensa de su tesis "Actividades biológicas de derivados del Ácido Araquidónico". Realizó estudios de doctorado en la University of North Texas, siendo el tema de su investigación "Biodegradación de componentes de petróleo contaminantes en aguas y suelos por bacterias del suelo"; en 1995, obtuvo el grado de Doctor en Ciencias, especialidad Biología. Ha recibido diversos reconocimientos y premios, siendo el más reciente el "Premio Nacional en Ciencia y Tecnología de Alimentos en la Categoría Profesional", que le fue otorgado en 2006 por la Industria Mexicana de Coca-Cola y CONACYT, promotores del citado concurso. Por su destacada labor científica, ha sido reconocida como Investigador Nacional Nivel I por el Sistema Nacional de Investigadores del CONACYT. Desde 1995 ha sido maestra de la Facultad de Ciencias Químicas (UACH) y su productividad científica incluye 17 artículos en revistas arbitradas. Además, ha editado más de cuatro libros y dirigido más de 65 tesis (licenciatura y maestría). La Dra. Nevárez pertenece a diversas sociedades científicas, citándose entre algunas de ellas: American Society for Microbiology, Society for Microbial Ecology y Sociedad Mexicana de Biotecnología y Bioingeniería.

Dr. Raúl Bautista Margulis. Egresó de la Unidad Docente Interdisciplinaria de Ingeniería y Ciencias Químicas de la Universidad Veracruzana. A partir de 1985 fue contratado por el Instituto de Investigaciones Eléctricas (IIE) como investigador de tiempo completo en el Departamento de Combustibles Fósiles de la División Fuentes de Energía. En 1990 obtuvo el grado de Maestro en Ciencias de la Combustión y Control de la Contaminación Ambiental y, en 1993, el grado de Doctorado en la Universidad de Sheffield-Inglaterra. Ese mismo año se reincorporó al IIE hasta julio de 1995 en el Depto. Sistemas de Combustión de la División Fuentes de Energía. De agosto de 1995 a abril del 2001, desempeñó el puesto de Jefe del Departamento de Ecología y Medio Ambiente en el Centro de Investigación en Materiales Avanzados (CIMAV). A partir de agosto del 2001, el Dr. Margulis fue contratado en la Universidad Juárez Autónoma de Tabasco (UJAT) como Profesor-Investigador en la División Académica de Ciencias Biológicas en la disciplina de Ciencias Ambientales. En el periodo abril 2003 - marzo 2007 desempeñó el cargo de Coordinador de Investigación y Posgrado de dicha División y, actualmente, pertenece al Sistema Nacional de Investigadores y al Sistema Estatal de Investigadores.\}

Luisa Idelia Manzanares Papayanopoulos. Cursó la carrera de Ingeniería Química en la Universidad Veracruzana, obteniendo su título en 1986. Realizó estudios de Maestría y Doctorado en Ciencias de la Combustión y Control de la Contaminación en la Universidad de Sheffield, Inglaterra en el período 1989-1995. Fue Investigadora Titular y Directora de la División de Ciencias Ambientales en el Centro de Investigación en Materiales Avanzados en el período 1996 - 2002. Del año 2003 a la fecha se desempeña como Directora de Auditorías Ambientales en la Procuraduría Federal de Protección al Ambiente de la SEMARNAT. 